\title{
APPELS ET CONTRE-APPELS DU « PEUPLE » À LA CANDIDATURE DE PAUL BIYA : AFFRONTEMENT PRÉÉLECTORAL, TENSIONS HÉGÉMONIQUES ET LUTTE POUR L'ALTERNANCE POLITIQUE AU CAMEROUN
}

\section{Jean-Marcellin Manga}

Karthala | « Politique africaine »

2018/2 $\mathrm{n}^{\circ} 150$ | pages 139 à 160

ISSN 0244-7827

ISBN 9782811125707

DOI 10.3917/polaf.150.0139

Article disponible en ligne à l'adresse :

https://www.cairn.info/revue-politique-africaine-2018-2-page-139.htm

Distribution électronique Cairn.info pour Karthala.

(C) Karthala. Tous droits réservés pour tous pays.

La reproduction ou représentation de cet article, notamment par photocopie, n'est autorisée que dans les limites des conditions générales d'utilisation du site ou, le cas échéant, des conditions générales de la licence souscrite par votre établissement. Toute autre reproduction ou représentation, en tout ou partie, sous quelque forme et de quelque manière que ce soit, est interdite sauf accord préalable et écrit de l'éditeur, en dehors des cas prévus par la législation en vigueur en France. Il est précisé que son stockage dans une base de données est également interdit. 


\section{LE DOSSIER}

JEAN-MARCELLIN MANGA

\section{APPELS ET CONTRE-APPELS DU «PEUPLE » À LA CANDIDATURE DE PAUL BIYA: AFFRONTEMENT PRÉÉLECTORAL, TENSIONS HÉGÉMONIQUES ET LUTTE POUR L'ALTERNANCE POLITIQUE AU CAMEROUN}

Cet article interroge les appels et contre-appels à la candidature de Paul Biya en tant qu'outils de participation politique. Les appels à la candidature de l'actuel président donnent à voir la construction de loyautés politiques derrière lesquelles se profilent divers arrangements clientélistes qu'imaginent les catégories dirigeantes en vue de stabiliser leurs positions hégémoniques. À l'opposé, les contre-appels mettent en évidence la manière dont, par des stratégies plurielles, leurs challengers $œ u v r e n t$ à éroder le symbolisme qui, depuis plus de trois décennies, institue Paul Biya comme le candidat naturellement élu à la fonction présidentielle. À travers l'intérêt porté à ces technologies de mobilisation électorale, l'on voudrait surtout mettre en relief la dimension processuelle des élections. Ainsi, l'importance accordée ici au moment préélectoral, plutôt qu'au temps court des opérations électorales, offre une porte d'entrée pour l'étude des dynamiques conflictuelles et contradictoires qui travaillent la société politique camerounaise.

Samedi 20 février 20161. De nombreuses personnalités du Rassemblement démocratique du peuple camerounais (RDPC), le parti au pouvoir, se sont donné rendez-vous à la place des fêtes de Bafoussam pour inciter le président Biya à solliciter un nouveau mandat à la présidence de la République, le cinquième depuis la libéralisation de la vie politique au début des années 1990. La cérémonie est solennelle, comme en témoigne sa retransmission en direct par Canal 2 International, l'une des plus importantes chaînes de télévision privée du pays. Parmi les personnes présentes, l'on distingue M. Niat Njifenji,

\footnotetext{
1. Je dois à Patrick Awondo l'idée de ce texte dont le projet remonte à 2016 et qu'initialement nous aurions dû rédiger conjointement. Dans sa forme actuelle, il est redevable à Alexandre Mbassi, Oscarine Mela et Gérard Amougou qui l'ont enrichi par les discussions régulières que nous avons eues. J'avoue ma dette à Merlin Ottou qui, en plus, s'est investi dans sa relecture attentive. Armand Leka mérite aussi d'être remercié pour ses lumières. Je suis enfin reconnaissant aux évaluateurs anonymes dont les commentaires sur les versions précédentes ont été inspirants.
} 
président du Sénat. À ses côtés figurent également d'autres illustres responsables politiques. À tour de rôle, devant une foule compacte et suivant une liturgie politique bien réglée, certains d'entre eux se relaient à la tribune pour magnifier les prouesses de leur champion, présenté comme le «candidat naturel» de leur formation politique dont, argumentent-ils, la présence est plus que jamais nécessaire à la tête du Cameroun. À l'issue de la cérémonie, un document intitulé «L'appel des fils et filles de l'Ouest» est publié2. Ce meeting de mobilisation et de soutien à $\mathrm{M}$. Biya en vue de la prochaine élection présidentielle s'inscrit dans le prolongement d'autres, initiés par des élites dans plusieurs régions à travers le pays et qui, tous, se clôturent par des appels à sa candidature.

En marge desdits appels, des contre-appels énonçant publiquement une opposition à cette nouvelle candidature potentielle fleurissent, portés, pour la plupart, par des leaders politiques de l'opposition et des acteurs associatifs ${ }^{3}$. Ces technologies de participation politique rendent compte de pratiques que les entrepreneurs politiques développent au cours du moment préélectoral. En choisissant cette entrée, l'on privilégie non pas ce qui se produit pendant et après le vote, mais davantage ce qui se joue avant ${ }^{4}$. On peut ainsi appréhender la manière dont se préparent les élections sur le long terme. L'avant-vote est ici pensé comme une conjoncture particulière caractérisée par une incertitude structurelle $^{5}$ qui offre aux acteurs du jeu politique de nouvelles marges de manœuvre. Il représente une fenêtre à partir de laquelle il est possible d'entrevoir les dynamiques conflictuelles et contradictoires qui travaillent la «société politique camerounaise ${ }^{6}$ ».

En prenant au sérieux les appels à la candidature de Paul Biya, cette analyse tente de mettre en évidence la manière dont ce mode d'expression et d'action donne à voir la construction de loyautés politiques à partir desquelles se jouent divers arrangements clientélistes qu'imaginent les catégories dirigeantes en vue de stabiliser leurs positions hégémoniques ${ }^{7}$. Au-delà de l'attention portée à ces appels, l'on voudrait également montrer de quelle manière, en produisant des contre-appels, différents leaders opposés à une

2. Voir «L'appel des fils et filles de l'Ouest», Jeune Afrique Économie, n 405, juillet-août 2016, p. 96-97.

3. Pour un exemple, voir le «Contre-appel national de la jeunesse», Le Jour, n² 2141, 10 mars 2016, p. 8.

4. J.-P. Olivier de Sardan (dir.), Élections au village. Une ethnographie de la culture électorale au Niger, tome 2, Paris, Karthala, 2015.

5. M. Dobry, Sociologie des crises politiques. La dynamique des mobilisations multisectorielles, Paris, Presses de la Fondation nationale des sciences politiques, 1986.

6. J.-F. Bayart, «La société politique camerounaise (1982-1986)», Politique africaine, n²2, 1986, p. 5-35.

7. L. Sindjoun, «Élections et politique au Cameroun: concurrence déloyale, coalitions de stabilité hégémonique et politique d'affection ", African Journal of Political Science, vol. 2, n 1, 1997, p. 89-129. 
nouvelle candidature de M. Biya s'emploient à éroder le symbolisme qui le pose comme le candidat naturellement élu à la charge présidentielle.

Appels et contre-appels mettent ainsi en évidence la manière dont s'expriment, aussi bien à l'intérieur du RDPC que dans les formations politiques concurrentes, les tensions entre forces rivales autour de la question du renouvellement du leadership. Si les catégories au pouvoir travaillent au maintien du statu quo, leurs challengers se mobilisent quant à eux pour faire advenir l'alternance au sommet de l'État. L'on ne peut entrevoir la véritable portée des rationalités à l'œuvre derrière ces discours si l'on fait fi des circonstances dans lesquelles ils sont produits. L'élaboration des appels à candidature s'ancre ainsi dans une dynamique ritualisée à la veille des scrutins présidentiels. Comme on le verra, leur fabrique et leur mise en visibilité montrent comment les élites au pouvoir s'investissent pour la réussite de ces mobilisations qui donnent à voir un travail de polissage, sans cesse renouvelé, de l'image du chef de l'État. En opposant aux paroles dithyrambiques, que véhiculent les appels, la radicalité des critiques qui sourd des contre-appels, les contestataires du régime récusent pour leur part le bilan de plus de trente ans de longévité. L'accent mis par ces opposants sur la durée de vie du régime et son incapacité à promouvoir l'«émergence» du pays sert surtout de prétexte pour dénoncer la mainmise d'élites vieillissantes sur la gestion des biens collectifs.

Dans cette contribution, l'on essaie de montrer que, bien qu'elle mette en lumière l'aptitude du régime à phagocyter plusieurs pans de la société, la production des appels et contre-appels informe surtout les désirs d'alternance et les demandes de changement qui travaillent, en profondeur, le champ politique national. Si la «naturalité» de la candidature de Paul Biya est remise en cause, c'est aussi parce que la croyance dans le déclin du régime semble entérinée par une partie de la classe politique qui y voit une opportunité. Cette conviction elle-même s'appuie sur l'idée qu'au regard de son âge avancé, l'actuel président ne saurait, pour longtemps encore, défier les lois de la biologie. La capacité du régime à se reproduire devenant sujette à caution, certains acteurs qui contestent une nouvelle candidature de l'actuel président en (ré-)interrogent la légitimité. À défaut d'une alternance fonctionnelle, d'autres tentent d'en contrôler certains enjeux ante-mortem.

L'étude s'appuie sur des entretiens menés auprès de leaders appartenant aussi bien au parti au pouvoir qu'à des formations politiques concurrentes. Une observation fine de certaines cérémonies politiques et d'émissions de débat en lien avec l'actualité des appels et contre-appels à la candidature de Paul Biya, retransmises par les chaînes de télévision publiques et privées entre 2016 et 2018, a été nécessaire. L'on exploite également des archives de presse. S'agissant de l'élection présidentielle de 2018 qui retient ici notre 
attention, si les appels à la candidature de Paul Biya sont manifestes dès la fin de l'année 2015 et se poursuivent jusqu'à ce que ce dernier déclare sa candidature le 13 juillet 2018, les contre-appels, eux, ne sont visibles qu'à partir de 2016 où leur effervescence s'étale sur trois mois. Entre janvier et mars, l'on a ainsi pu en dénombrer sept. En plus de contester la candidature de Paul Biya en 2018, ils réagissent aux appels de certains de ses partisans l'invitant à anticiper la tenue du scrutin présidentiel eu égard à la pluralité des échéances électorales censées se tenir cette année. Cette comptabilité ne prend pas en compte les déclarations opposées à la candidature du président Biya parues sous la forme d'interviews dans la presse ou sur les réseaux sociaux. Sur la même période, le quotidien gouvernemental Cameroon Tribune publiait 78 appels à la candidature de M. Biya.

Notre recherche considère tout d'abord l'inscription historique des appels à candidature. Elle examine, par la suite, leur matérialité, leurs porteurs et la prétention de ces derniers à parler «au nom du peuple». La fabrique d'un appel retient aussi l'attention. L'on interroge, pour finir, les contre-appels en insistant, à la fois, sur leur ossature, leurs auteurs et la portée de leurs discours.

\section{LES RACINES D'UNE PRATIQUE}

Les appels à candidature font référence à des pratiques à travers lesquelles des acteurs individuels ou, plus généralement, collectifs affichent leur loyauté à Paul Biya. Par ces actes, ils l'exhortent à se porter à nouveau candidat à la magistrature suprême. S'ils connaissent une forte publicité sous son régime, les appels à candidature actualisent néanmoins une pratique politique qui prospérait déjà sous Ahmadou Ahidjo, le premier président ${ }^{8}$. Aux côtés d'autres types de langage ${ }^{9}$, ils rendent compte de la manière dont certains dispositifs ayant nourri le régime de ce dernier alimentent activement celui de son successeur. Il importe tout de même de noter que, sous Paul Biya, ils n'ont pas toujours eu le vent en poupe. Dans la fièvre des élections présidentielles de 1992 et 1997, alors que le pouvoir faisait face à une vive contestation de l'opposition, il n'y a pas eu d'appels. Il faut attendre la veille du scrutin présidentiel de 2004, dans des circonstances (re)devenues favorables au parti au pouvoir, pour les voir (à nouveau) se déployer.

8. Union nationale camerounaise, Troisième congrès ordinaire de l'Union nationale camerounaise. Congrès de la maîtrise, Paris, Groupe média international, 1982, p. 346-347.

9. R. L. Aka'a, Les motions de soutien au Cameroun: contribution à l'étude d'un mode particulier de participation politique, Mémoire de Master en sociologie politique, Yaoundé, Université de Yaoundé, 2013. 
Appels et contre-appels du «peuple» à la candidature de Paul Biya

Bien que la production des appels à la candidature de Paul Biya survienne aussi au cours de l'année durant laquelle les élections ont lieu, leur expression est davantage manifeste en amont. Pour celle de 2018, c'est le 6 novembre 2015, à l'occasion du 33e anniversaire de l'accession du président Biya au pouvoir, que les premiers appels à sa candidature ont été lancés avant de gagner en acuité les années suivantes. La diffusion des appels à candidature coïncide avec une période de "crue politique ${ }^{10}$ », entendue comme un moment de la vie politique nationale antérieur aux élections qui, en raison d'enjeux décisifs, gagne en intensité et est perçu, par des entrepreneurs politiques concurrents, comme une opportunité à saisir. Ainsi, entre 2006 et 2008, plusieurs appels à candidature se sont fait entendre afin de justifier la modification de la constitution visant à lever le verrou de la limitation du mandat présidentiel qui aurait mis Paul Biya hors course pour l'élection de 2011. Dans une perspective similaire, le contexte sécuritaire de la guerre contre Boko Haram donne aussi l'occasion aux fidèles du président de l'appeler à se porter à nouveau candidat à la présidentielle de $2018^{11}$.

\section{LA STRUCTURE DES APPELS}

Dans sa forme, un appel à candidature est un document qui a fait l'objet d'une publication officielle. Ses principaux supports de diffusion sont le Cameroon Tribune, le quotidien gouvernemental, et l'Action, l'organe de presse du RDPC. Pour les élections présidentielles de 2011, la Société de presse et d'éditions du Cameroun (Sopecam), établissement à capitaux publics a, en 2009, mis sur le marché Paul Biya, l'appel du peuple. The People's Call12, un recueil en cinq tomes réunissant des textes de personnalités pressant le président Biya à se porter candidat.

La structure des appels à candidature fait apparaître sept éléments au moins. On a, en premier lieu, une antienne d'ouverture dans laquelle les porteurs déclinent leur identification. En les parcourant, on peut régulièrement constater la référence au Nous: «Nous, membres du Comité central du RDPC, Député à l'Assemblée nationale, Maire, Présidente et Présidents, forces vives,

\footnotetext{
10. C'est à A. Leka que nous empruntons cette expression. Voir ses développements au cours de l'émission «Scènes de presse» du 13 mais 2018 consacrée à l'unité nationale: < https://www.youtube. com/watch?v=p71FBzdVWVE>, consulté le 9 juin 2018.

11. M.-E. Pommerolle, «Les violences dans l'Extrême-Nord du Cameroun: le complot comme outil d'interprétation et de luttes politiques », Politique africaine, $\mathrm{n}^{\circ} 138$, 2015, p. 163-177. Consulter aussi utilement P. E. Batchom, «La guerre du peuple: de la popularisation de la guerre contre Boko Haram au Cameroun", Études internationales, vol. 47, n²-3, 2016, p. 285-304.

12. P. Biya, L'appel du peuple, Yaoundé, Sopecam, 2009.
} 
sympathisants militantes et militants des sous-sections OFRDPC (Organisation des femmes du Rassemblement démocratique du peuple camerounais), OJRDPC (Organisation des jeunes du Rassemblement démocratique du peuple camerounais) et RDPC de Bamboutos Centre II... ${ }^{13}$ ». Par cette tournure, les acteurs qui promeuvent les appels entendent affirmer leur légitimité à parler au nom du plus grand nombre. En prêtant attention à l'identification des porteurs des appels à la candidature de Paul Biya, on se retrouve en présence d'une diversité de profils qui autorise à penser l'existence d'une compétition entre groupes pour afficher son soutien au président. Outre des militants du RDPC, la présence des leaders de partis alliés est aussi observable.

Les appels à candidature exaltent cependant davantage les liens d'appartenance communautaire. C'est ce qu'indique l'appel de l'Ouest auquel il a été fait allusion en introduction et sur lequel nous reviendrons plus loin. On peut aussi le vérifier à travers les appels du Sud ${ }^{14}$, de la Lekié15 et bien d'autres $^{16}$. Parfois, cette appartenance s'accompagne de la référence à une catégorie professionnelle. Dans les deux configurations, ils rendent compte des dynamiques de fragmentation de la société politique camerounaise ${ }^{17}$. Les politiques n'ont donc pas l'exclusivité des appels. Différents corps professionnels ayant un ancrage aussi bien national qu'international invitent également Paul Biya à présenter à nouveau sa candidature.

Parmi les personnalités extérieures au champ politique qui appellent vigoureusement celui-ci à se représenter, celles issues des milieux universitaires occupent une place de choix ${ }^{18}$. À l'orée de l'année 2004, année au cours de laquelle eut lieu la troisième élection présidentielle depuis le retour au multipartisme, quelque 200 universitaires publièrent l'« Appel de l'intelligentsia à Paul Biya ${ }^{19}{ }^{\prime}$, scénario qui allait se répéter dans la perspective du scrutin suivant, en 2011. Si de telles initiatives peuvent surprendre, au Cameroun, le fait que des enseignants d'université ajoutent leurs voix au chœur de celles qui appellent à une nouvelle candidature un président de la République ne

13. «Appels à la candidature de Paul Biya. La section RDPC de Bamboutos-Centre II», Cameroon Tribune, $\mathrm{n}^{\circ} 11073 / 7272,12$ avril 2016, p. 11.

14. «Présidentielle 2018. Le Sud a choisi son candidat: c'est Paul Biya», Cameroon Tribune, $\mathrm{n}^{\circ} 11603 / 7802,25$ mai 2018, p. 10-11.

15. «L'Appel républicain de la Lékié », Cameroon Tribune, n 11597/7796, 16 mai 2018, p. 11.

16. «Nord-Ouest, Sud-Ouest, Ouest, Littoral. Paul Biya candidat des chefs traditionnels», Cameroon Tribune, $\mathrm{n}^{\circ} 11575 / 7774,11$ avril 2018, p. 4-6.

17. A. Leka Essomba, Les dynamiques de la citoyenneté en société postcoloniale. Contribution à une sociologie des désordres identitaires au Cameroun, Mémoire de maîtrise en sociologie, Yaoundé, Université de Yaoundé I, 2001.

18. P. Akana, «Les universitaires dans la cité: sur les enjeux de la prise de parole dans l'espace public au Cameroun» [en ligne], <https://www.codesria.org/IMG/pdf/Parfait_Akana.pdf?1321/ a1ff34b63e84848c3ce41939f103827aec0a2278>, consulté le 12 septembre 2018.

19. «L'appel de l'intelligentsia à Paul Biya», Cameroon Tribune, 27 janvier 2004, p. 5. 
relève pas de l'inédit. En 1975, par exemple, certains d'entre eux prièrent Ahidjo de se représenter à la présidentielle ${ }^{20}$. Cherchant à conférer à Paul Biya une onction de légitimité supplémentaire au lendemain de la démission de son prédécesseur, des universitaires lui apportèrent leur caution en $1983^{21}$.

Pour en revenir à la structuration des appels, après l'antienne d'ouverture, vient l'interpellation du destinataire, le président de la République vers qui monte cette oraison. Dans le texte de leur appel, les «artistes du Sud» en fournissent une illustration: "Nous, artistes du Sud, et tous Arts confondus, adressons à Son Excellence Monsieur Paul Biya, président de la République...22 ». Un troisième moment est repérable. Il s'agit d'une justification de l'initiative enclenchée vantant, très souvent, les faveurs dont le pays, la région, la catégorie professionnelle concernée, etc. a bénéficié de la part du président au cours de son mandat précédent ${ }^{23}$. À ce moment, il est encensé pour la qualité de son leadership, ses «vertus politiques» (doigté, clairvoyance, maîtrise et sagesse ${ }^{24}$ ) ou encore ses «grandes réalisations».

La quatrième étape met en avant les remerciements adressés au président. Ses fidèles insistent sur la réalisation d'infrastructures matérielles qui permettent des opportunités de développement et la promotion - ou le maintien ${ }^{25}$ - à d'importantes fonctions d'un ou de plusieurs «digne(s) fils de leur terroir ». Après lui avoir réaffirmé leur «soutien indéfectible» dans la cinquième étape, la sixième engage fermement le président à solliciter un nouveau mandat en lui garantissant une «victoire totale». L'énumération des signataires, dont l'effectif est généralement important ${ }^{26}$, clôt l'appel. Si l'on analyse la rhétorique à travers laquelle les élites appellent M. Biya à proposer, une fois de plus, sa candidature à la présidence de la République, une des constances qui émerge est l'omniprésence de la référence au peuple.

\section{METTRE LE PEUPLE EN ORDRE : UN ART POLITIQUE}

Cette référence au peuple doit beaucoup à la présence, parmi les élites, d'élus dont la légitimité repose sur leur investiture par les populations. Au cours du meeting de Bafoussam, lorsqu'il prend la parole au nom des maires

\footnotetext{
20. Cameroon Tribune, $\mathrm{n}^{\circ} 172,28$ janvier 1975, p. 2.

21. Cameroon Tribune, $\mathrm{n}^{\circ} 2759,28$ août 1983.

22. Cameroon Tribune, $\mathrm{n}^{\circ} 11073 / 7272$, op. cit., p. 10.

23. Cameroon Tribune, $\mathrm{n}^{\circ} 11575 / 7774$, op. cit., p. 6

24. Cameroon Tribune, $\mathrm{n}^{\circ} 11597 / 7796$, op. cit., p. 11.

25. Ibid., p. 6.

26. On a par exemple dénombré 823 noms dans «La déclaration d'Akonolinga». Voir Cameroon Tribune, $\mathrm{n}^{\circ} 11602,24$ mai 2018, p. 9.
} 
et des conseillers municipaux de la région de l'Ouest, Charles Tchomtchoua Djadjo, le maire de Banwa, achève son propos en mettant en exergue ce pouvoir: «[...] nous le [Paul Biya] prions de bien vouloir accepter d'être le candidat des populations de la région de l'Ouest à la prochaine élection présidentielle ${ }^{27}$ ». Un son de cloche similaire ressort du discours que Théodore Datouo, vice-président de l'Assemblée nationale, prononce à sa suite, avec ceci de particulier qu'il y fait explicitement référence au parti: «Les filles et les fils de notre région [...] sont tous unis en rangs serrés derrière vous, sous la conduite des lieutenants que vous avez vous-mêmes désignés sur le terrain ${ }^{28}$ ».

L'allusion que l'honorable Théodore Datouo fait aux lieutenants désignés par Paul Biya, le président de la République, agissant ici en tant que président national du RDPC, est riche de sens. Elle renseigne sur la façon dont la pensée et l'action des élus sont encadrées en faisant valoir le respect de la discipline du parti. Par cette opération, le parti affirme sa préséance sur les élus et se pose comme une interface entre eux et la base.

Les lieutenants qu'évoque Théodore Datouo représentent l'administration du parti au sommet de laquelle se trouve le comité central, son organe directionnel, représentée au rassemblement de Bafoussam par son secrétaire général, Jean Nkuete. Dans ce meeting, l'administration étatique est aussi présente. Le rituel protocolaire confirme la relégation des élus locaux au second plan. À titre d'illustration, la parole ne sera donnée aux représentants des maires et des conseillers municipaux qu'après que, dans l'ordre, le délégué du gouvernement, le président de la section RDPC de la Mifi-Centre ${ }^{29}$, le roi des Bayangam et le représentant des opérateurs économiques eurent décliné leurs propos. La préséance de l'administration du parti sur les élus est également attestée par les prérogatives dont jouit celui qui occupe la fonction de chef de délégation permanente du comité central du RDPC pour une circonscription.

Cette fonction ne doit pas seulement être vue comme un dispositif qu'utilise le parti pour contrôler ses élus. Elle est aussi la médiation par laquelle l'administration publique s'allie à celle du parti pour les cantonner à une position subalterne. Ainsi, il est des cas où le chef de la délégation permanente du comité central du RDPC a également un portefeuille au sein du gouvernement. Henri Eyebe Ayissi, le chef de la délégation permanente du comité central du RDPC pour le département de la Lekié, constitue, de ce

27. «L'appel des fils et filles de l'Ouest», art. cité, p. 75.

28. Ibid.

29. La Mifi est un des huit départements que comporte la région de l'Ouest. Dans le découpage administratif du RDPC, la Mifi-Centre est une section dont la centralité s'explique par son ancrage géographique à Bafoussam, le chef-lieu du département. 
Appels et contre-appels du «peuple» à la candidature de Paul Biya

point de vue, un exemple parlant. Cette concentration de pouvoir engendre souvent une exacerbation de la conflictualité et une concurrence entre élites pour la représentation. Pour en limiter les incidences fâcheuses, la hiérarchie du parti procède alors à des arbitrages qui, s'ils réussissent à définir un consensus entre parties antagonistes, favorisent surtout l'émergence d'un «homme fort» dans l'arène politique locale.

Ces antagonismes ne doivent néanmoins pas tromper. Ces élites, qu'elles soient bureaucratico-politiques ou élues, lorsqu'elles sont acquises au RDPC, travaillent à construire la légitimité de Paul Biya. Cet effort, qui participe véritablement d'un «rite d'institution ${ }^{30}$ », se fait à travers une série d'opérations. Le point de départ consiste à diffuser l'idée de sa candidature dans l'espace public. Si ces élites déploient une telle énergie pour asseoir la légitimité de leur champion, c'est que l'idée de la naturalité de sa candidature ne va pas de soi au sein de sa propre formation politique. À l'intérieur du RDPC, en effet, émerge une ligne de front, de plus en plus visible, qu'animent des élites militantes et des militants de la base qui expriment leur opposition au déficit d'alternance à la tête du parti. Deux modalités de cette dissidence, qui s'insèrent dans un courant réformateur, sont observables.

La première et la plus explicite de ces deux modalités, celle dite «des modernistes ${ }^{31} »$, a longtemps été associée à une ambition de changement promue par Mila Assouté, un ancien membre du comité central du parti qui a par ailleurs exercé comme député durant près d'un quart de siècle. Suite au rejet d'une série de réformes qu'il avait proposées pour rénover le parti, il rejoint l'opposition en 2004. Aujourd'hui exilé en France, il se porte candidat en 2004 également à l'élection présidentielle sous les couleurs du Ralliement au changement pour la nouvelle République (RCNR). Sa candidature est toutefois rejetée comme le sera également celle qu'il présente en 2011, cette fois sous la bannière du Rassemblement démocratique pour la modernité du Cameroun (RDMC), la nouvelle dénomination du RCNR. D’autres profils incarnent cette tendance. Charles Messanga Nyamding, membre titulaire du comité central et figure publique reconnue pour ses prises de position sévères envers les élites du parti, est de ceux-là. En 2014, il avait d'ailleurs déjà écopé d'un blâme. Saint Éloi Bidoung qui, pour avoir récemment fait connaître son désir de se porter candidat à la présidence du RDPC, a essuyé des menaces d'expulsion du parti en fait également partie.

30. P. Bourdieu, «Les rites comme actes d'institution», Actes de la recherche en sciences sociales, $\mathrm{n}^{\circ} 43$, 1982, p. 58-63.

31. P. Adjedja, Luttes intrapartisannes au Cameroun. Cas du conflit autour de la "modernisation» du Rassemblement démocratique du peuple camerounais, Mémoire de maîtrise en sociologie politique, Yaoundé, Université de Yaoundé, 2008. 
Cameroun, l'État stationnaire

Le second type de dissidence est tacite et prend la figure d'un parricide. Il est généralement le fait d'anciens proches collaborateurs du président accusés de manœuvrer pour l'évincer, alors même qu'ils avaient, pendant un temps, été présentés à l'opinion comme des héritiers potentiels. Au sein du parti, Marafa Hamidou Yaya est sans doute la personnalité qui, actuellement, incarne le mieux ce type de profil. Ancien baron du régime Biya et membre du bureau politique, il est, depuis 2012, incarcéré dans une cellule du secrétariat d'État à la défense (Sed), officiellement pour «coaction de détournement des deniers publics». De façon implicite, il est «coupable» d'avoir suggéré à Paul Biya de ne pas se représenter en 2011 et de s'être positionné comme un potentiel rival. Le sort réservé à Marafa comme, avant lui, à Titus Edzoa ou, plus récemment, aux membres de la nébuleuse « $\mathrm{G} 11^{32}$ » en dit long sur la manière dont ce type de rébellion est marginalisée. Ici, le parti active des mécanismes d'État pour châtier les «dissidents ${ }^{33}$ ».

Du coup, face à la montée en visibilité d'une opinion partisane hostile à la pérennisation du prince à la tête du parti qu'expriment des personnalités porteuses de ce qu'à la suite de Leka Essomba, on pourrait nommer un «charisme de remplacement ${ }^{34}$ », la construction, par certaines élites, d'un discours visant à montrer que «c'est le peuple» qui appelle M. Biya à se porter candidat prend tout son sens.

\section{PROdUIRE UNe COUTUME POLITIQUe: LeS COULISSES D'UN APPEL}

Pour avoir une idée de la cuisine d'un appel à candidature, il faut esquisser une description des principales étapes qui interviennent dans l'élaboration de ce rituel partisan. Grosso modo, sa construction fait intervenir deux étapes cruciales.

32. Alimentée par des rumeurs circulant dans des salons huppés, puis popularisée par la presse locale, l'appellation «G11» a fleuri dans le sillage de l'élection présidentielle de 2011 pour désigner un collectif de responsables occupant des positions de premier plan au sein du gouvernement - aujourd'hui embastillés dans le cadre de l'«Opération Épervier» - à qui l'on a prêté l'ambition de vouloir succéder au président Biya.

33. A. Leka Essomba, «Gouverner et punir. Justice, corruption et récit de pouvoir au Cameroun», Fréquence Sud. Revue camerounaise de sciences de l'information et de la communication, $\mathrm{n}^{\circ}$ 23, 2017, p. 157-177.

34. Ibid., p. 159. 
Appels et contre-appels du «peuple» à la candidature de Paul Biya

\section{De l'ombre...}

La première, considérée comme préparatoire, est faite de réunions assez fréquentes. Il s'agit de conciliabules initiés par un «fils du terroir», dont la légitimité est admise du fait de l'accréditation qu'il a reçue du parti. Dans la Lekié, «c'est le ministre Eyebe Ayissi... ${ }^{35}$ », agissant en sa qualité de chef de la délégation permanente départementale du comité central du RDPC pour cette subdivision «qui, plusieurs fois, a été à la tête de telles initiatives ${ }^{36}$ ». Pour les élites bureaucratico-politiques, ces rencontres préparatoires se déroulent le plus souvent dans les bureaux administratifs du fait des fonctions qu'occupent leurs membres au sein du gouvernement ou dans la haute administration publique. Plusieurs concertations relatives à la préparation de l'Appel de Monatélé se seraient ainsi tenues dans les locaux du ministère du Contrôle supérieur de l'État (Consupe) au moment où M. Henri Eyebe Ayissi y était ministre ${ }^{37}$. Marqués du sceau d'une grande discrétion, ces conciliabules réunissent des membres du parti. L'appartenance à celui-ci n'est pour autant pas le seul critère déterminant. L'affinité personnelle avec l'organisateur est aussi une variable décisive dans l'association à cette dynamique de groupe qui valorise une proximité ethnique. Le choix porté par l'organisateur de ces conciliabules sur les individus présents se justifie également par les alliances sur lesquelles leur proximité et celle de leurs réseaux sont susceptibles d'ouvrir. Ces alliances politiques s'expliquent par des antagonismes intrapartisans, souvent ouverts, au niveau local. Le désir de s'allier certaines catégories stratégiques est un autre élément qui permet de comprendre la présence, lors de ces concertations, de personnes ou de groupes ne partageant pas forcément une affinité avec le parti.

La rédaction du document qui officialise l'appel est faite à l'ombre des regards par son initiateur. Dans certaines circonstances, du fait de différends avec une personnalité éminente du même «fief», il est arrivé que celui-ci refuse que le nom d'un adversaire figure parmi les signataires ${ }^{38}$. Ainsi, la rédaction et la signature du texte de l'appel font l'objet de rivalités internes. Le chef de délégation permanente du comité central investi par le parti peut, par conséquent, disqualifier ses concurrents et, dans le même temps, qualifier ses soutiens. Cette compétence autorise à le définir comme un big man $^{39}$ dont

35. Entretien avec un leader de l'Association des étudiants de la Lekié (Aedele), militant du RDPC, Yaoundé, 22 décembre 2017.

36. Ibid.

37. Ibid.

38. Entretien avec un responsable du parti, Yaoundé, 12 décembre 2017.

39. J.-F. Médard, «Le "big man” en Afrique: esquisse d'analyse du politicien entrepreneur», L'année sociologique, vol. 42, 1992, p. 167-192. 
l'influence est renforcée par la position qu'il occupe au sein du gouvernement ou de l'administration publique. C'est à travers ces multiples casquettes qu'il assoit sa domination dans un environnement où le parti surveille étroitement ses cadres:

«Des listes de présence circulent... Il y a des secrétaires qui sont chargés de faire circuler ces listes où les chefs traditionnels, les chefs religieux, les membres du bureau politique mentionnent leurs noms. La proposition des listes vient du comité central. C'est-à-dire, il y a le nom, il y a la qualité, il y a le poste que vous occupez dans le RDPC et puis, il y a une autre marge qui présente votre fonction hormis le cadre du RDPC, et puis il y a la signature ${ }^{40} \gg$.

En dépit de ce dispositif de surveillance, des élites opposées à certaines actions entreprises par le parti parviennent souvent à manifester leur désaccord. En 2008, voyant d'un mauvais œil une possible (re)candidature de Paul Biya à l'élection présidentielle de 2011, M. Henri Engoulou, ex-ministre délégué aux Finances en charge du budget, avait ainsi refusé, au cours d'un meeting politique tenu à Monatélé, d'apposer sa signature sur une déclaration de soutien. Certains ont cru voir dans cette attitude l'origine de sa disgrâce survenue deux ans plus tard ${ }^{41}$.

\section{... à la pénombre}

Dans la deuxième étape se joue un intense travail administratif au sein du parti, sous l'œil, toujours vigilant, du comité central. À la base de cet investissement, se trouve, une fois de plus, le chef de la délégation permanente départementale du comité central du RDPC. C'est lui qui initie les lettres d'information puis, plus tard, d'invitation qui seront distribuées aux présidents des sections. À leur tour, ceux-ci convient les présidents de soussections. C'est à ces derniers que revient la charge d'inviter les présidents des comités de base en prenant soin de leur demander de sensibiliser les militants de sorte qu'ils se tiennent prêts à effectuer le déplacement, le moment venu, vers le site retenu pour la mobilisation. Cette structure épouse les contours de l'organisation hiérarchique du parti.

40. Entretien avec un cadre du parti, Yaoundé, 12 décembre 2017.

41. Ibid. Voir aussi L.-M. Kakdeu, «Henri Engoulou, mort pour avoir rejeté le griotisme politique» [en ligne], Cameroon Voice, 14 mai 2014, <http://www.cameroonvoice.com/news/article-news-15110. html $\gg$, consulté le 4 mars 2018. 
Si une partie des fonds nécessaire à la tenue de tels regroupements est prélevée dans les caisses du parti, les élites locales apportent également leur pierre à l'édifice en se cotisant. Le taux exigé dépend, le plus souvent, du niveau de responsabilité occupée au sein du parti ou de l'administration publique. La plupart du temps, c'est le chef de la délégation permanente départementale du comité central pour le RDPC qui répartit l'argent réuni. Situés en haut de la pyramide, les présidents de section se taillent la part du lion. Ils doivent néanmoins en redistribuer une quantité à leurs camarades en responsabilité dans d'autres branches du parti. Les sommes partagées peuvent être utilisées pour prendre en charge la logistique, le transport et/ou la restauration des militants. Des gratifications sont prévues. Les «motivations» promises dépendent, là encore, de la position occupée et varient selon la nature et l'envergure de l'événement.

Les militants peuvent percevoir 1000, 2000 ou 3000 francs CFA par personne. À chacun des présidents des comités de base, on peut accorder 10000 francs CFA. Quant aux présidents de sous-section et de section, ils obtiennent une somme comprise entre 15 et 20000 francs CFA. S'agissant des premiers, au-delà de ces frais, il arrive que l'on mette à leur disposition des fonds supplémentaires - jusqu'à 300000 francs CFA, selon une source ${ }^{42}$. Les présidents de sous-section disposent d'une marge de liberté dans la gestion des sommes qui leur sont allouées. L'autonomie dont ils jouissent sert pour la mobilisation des effectifs et n'est pas sans conséquences sur le fonctionnement du parti ${ }^{43}$. En décidant, dans la répartition, qu'il faut en donner plus à certains et peu, ou rien, à d'autres, leur «arbitrage alimente souvent des conflits... Il y a des clans qui se créent entre les différents membres, les partisans de A devenant les ennemis de B. Du coup, on assiste à des scènes où des partisans peuvent boycotter un événement du parti ou venir organiser du désordre ${ }^{44}$ ». L'analyse de la construction d'un appel à candidature fait ainsi apparaître deux phases décisives que sont les tractations engagées dans le cadre des préparatifs et l'investissement administratif du parti selon des usages codifiés.

42. Entretien avec un cadre du parti, Yaoundé, 12 décembre 2017.

43. Leur action se rapproche ainsi de celle des présidents de regroupements de chauffeurs dont Sydi Cissokho étudie le positionnement en contexte électoral au Sénégal et qu'il décrit comme «de véritables intermédiaires entre les partis et la population chauffeurs des gares » bénéficiant d'une forte marge de manœuvre. S. Cissokho, «L'enchâssement du moment électoral dans l'ordinaire syndical. Le Syndicat national des travailleurs du transport sénégalais en campagne», in J. Gervais et G. Courty (dir.), Le lobbying électoral. Groupes en campagne présidentielle, Lille, Presses universitaires du Septentrion, 2016, p. 167.

44. Entretien avec un responsable du parti, Yaoundé, 4 juin 2018. 


\section{INVENTER UN NOUVEAU LANGAGE POLITIQUE}

En réaction aux appels à la candidature de Paul Biya à la présidentielle de 2018 soutenue par les élites, des acteurs politiques et associatifs opposés à ces initiatives ont lancé des contre-appels. Si, comme cela s'est vu avec les appels, ils prétendent eux aussi parler «au nom du peuple», le point de vue des jeunes et la référence à la jeunesse y sont manifestes.

\section{Chair et artisans des contre-appels}

Les contre-appels à la candidature de Paul Biya renvoient à des documents dont la production est intimement liée à celle des appels et dans lesquels ceux qui les publient prennent ouvertement position contre une nouvelle candidature du président. Ces écrits peuvent revêtir différentes formes. Il peut, par exemple, s'agir d'une lettre ouverte, d'une déclaration argumentée ou, plus rarement, d'un tract ${ }^{45}$. Ceux-ci sont relayés dans des supports de presse favorables à l'opposition et sur les réseaux sociaux numériques. Il est aussi des cas où, sans en porter explicitement la formulation, certains documents, par leur contenu, épousent la philosophie des contre-appels décrite en amont.

Contrairement à ce que pourrait laisser penser leur effervescence en 2016, les contre-appels sont déjà repérables à la veille de certains scrutins présidentiels antérieurs à 2018. En 2004, par exemple, au lendemain du Forum de Dschang organisé par le ministère de l'Enseignement supérieur (Minesup) et auquel ils avaient pris part, des leaders étudiants découvrent un communiqué qu'ils auraient signé, et dans lequel il est fait mention de leur satisfaction vis-à-vis de l'action du président de la République. Mécontents, et un peu en réaction à l'appel à la candidature de Paul Biya lancé par leurs enseignants, certains d'entre eux s'en distancient en publiant «L'appel des 1000 » où ils se prononcent contre une nouvelle candidature de Paul Biya au scrutin présidentiel de $2004^{46}$. Deux ans avant l'élection présidentielle de 2011, le Front de la jeunesse contre la candidature de Paul Biya en 2011

45. Ainsi, par exemple, de celui du Collectif des organisations démocratiques et patriotiques des Camerounais de la diaspora (Code) où l'on pouvait lire ceci: «Paul Biya, c'est ton tour de dégager» ou encore «Paul Biya dégage».

46. Entretien avec un ancien leader estudiantin de l'université de Yaoundé II-Soa, Yaoundé, 29 janvier 2018. Voir aussi «Appel de la jeunesse. Les leaders estudiantins parlent. Les manœuvres du Minesup dénoncées », Mutations, n 1130, 15 avril 2004, p. 3. Sur le même sujet, voir X. Deutchoua, «Appel des intellectuels: 1000 étudiants répondent à leurs enseignants» [en ligne], <http://www. cameroon-info.net/reactions/appel-des-intellectuels-1000-etudiants-repondent-a-leurs-enseignants87450.html>, consulté le 12 septembre 2018. 
voit le jour lors d'une réunion à Mvomeka'a, le village natal du président de la République, dans le cadre de la célébration du $44^{\mathrm{e}}$ anniversaire de la fête de la jeunesse.

Les contre-appels sont toutefois particulièrement nombreux et agressifs dans la perspective de la présidentielle de 2018. Vice-président de la section des jeunes militants du premier parti d'opposition, le Front social démocrate ou Social Democratic Front (SDF), pour la région du Centre, Parfait Mbvoum rédige par exemple le «Contre-appel public des vrais fils du Sud contre le président Biya». Originaire comme ce dernier de cette région, il s'y évertue à démontrer que «les vrais fils du Sud ne sont pas d'accord ${ }^{47}$ ». Il «montre les limites de ce que c'est que le $\mathrm{Sud}^{48}$ » et attire l'attention sur «les risques que le Sud encourt à avoir encore $\mathrm{M}$. Biya comme président ${ }^{49}{ }$. Un de ces périls, croit-il percevoir, a trait au fait qu'à son départ, cette aire géo-culturelle pourrait être tenue responsable des difficultés auxquelles fait face le pays ${ }^{50}$.

D'un point de vue général, la structuration des contre-appels rejoue la démarche qui inspire les appels. Ils s'ouvrent par un préambule où ce qui est aussi en jeu c'est de «faire croire ${ }^{51}$ » que l'on est investi d'une mission de représentation. Ils pointent ensuite un doigt accusateur sur les élites dirigeantes, incriminant leur gouvernance. Cette deuxième articulation donne à voir une stratégie discursive où, sur fond d'objection, leurs artisans répondent aux justifications que les tenants des appels donnent pour motiver leur adhésion à Paul Biya. Alors que les derniers mettent davantage l'accent sur les «grandes réalisations», les premiers insistent sur ce qui, depuis l'avènement de ce dernier au pouvoir, ne va pas. Du coup, les porteurs des contre-appels égrainent un chapelet de déficits dont ils jugent les élites, au premier chef le président de la République, responsables. Ils lui reprochent $\mathrm{d}^{\prime}$ «avoir conduit le pays au bord du gouffre ${ }^{52}$ » avec la complicité active des militants du RDPC. La corrélation que ces acteurs établissent entre la longévité de Paul Biya au pouvoir, la gérontocratisation de la vie politique et la dégradation des conditions de vie des populations, avec une insistance particulière sur les jeunes, amène à réinterroger la responsabilité des aînés sociaux dans ce qu'ils nomment l'«échec du renouveau ${ }^{53}$ » au Cameroun.

47. Entretien avec Parfait Mbvoum, Yaoundé, 14 décembre 2017.

48. Ibid.

49. Ibid.

50. Ibid.

51. P. Champagne, Faire l'opinion. Le nouveau jeu politique, Paris, Minuit, 1990.

52. <http://petitionpublique.fr/PeticaoVer.aspx?pi=P2016N48595>, consulté le 5 juin 2018.

53. D. E. Atangana, «Cameroun: Contre l'appel de la Lékié!!! : CAMEROON» [en ligne], Camer.be, <http://www.camer.be/49700/30:27/cameroun-contre-lappel-de-la-lekie-cameroon.html>, consulté le 8 juin 2018. 
Cameroun, l'État stationnaire

Leur propos travaille ainsi à transcender le constat qui, dans le discours institutionnel ${ }^{54}$, établit une corrélation entre délinquance et jeunesse pour insister sur l'idée de la prégnance, dans le contexte de la société camerounaise actuelle, de ce que je qualifierais de «délinquance sénile». C'est sans doute pour cette raison que, dans le contenu des contre-appels qu'ils rédigent, leurs auteurs mettent l'accent sur la conscience de la jeunesse.

\section{Au nom des jeunes?}

Les porteurs des contre-appels sont souvent des jeunes gens qui militent dans des organisations associatives. Cela est vérifiable autant pour le «Contreappel de la Lekié55 ${ }^{5}$ que pour celui ayant un retentissement national «de la jeunesse consciente et républicaine ${ }^{56}$ ». Dans ces deux contre-appels, tous prétendent parler au nom des jeunes. À la question de savoir au nom de quelle jeunesse ils parlent précisément, Denis Atangana, l'un des inspirateurs du contre-appel de la Lekié qui a activement milité au sein du Mouvement pour la renaissance du Cameroun (MRC) ${ }^{57}$ et est, par ailleurs, le promoteur du Cercle de réflexion et d'action pour le développement (Cerad), une association investie dans des activités citoyennes, répond que c'est au nom de la jeunesse consciente et républicaine de ce département que cette décision a été prise:

«On n'a pas dit qu'on a fait l'appel au nom de tous les jeunes de la Lekié. Nous, on a fait notre contre-appel [au nom de la] jeunesse consciente et républicaine. Et, on sait qu'il y a plusieurs types de jeunesse. Il y a des jeunes qui boivent les bières. Il y a des jeunes qui vont à l'église... Il y a quand même aussi des jeunes qui sont conscients et qui ont l'esprit de la République. Et c'est à ce titre-là qu'on a signé notre contre-appel ${ }^{58}$ ».

Établissant cette distinction, les jeunes qui portent cette initiative affirment leur singularité en se démarquant de ce qui leur apparaît comme une jeunesse disqualifiée. S'ils font allusion aux jeunes qui «boivent la bière» et à «ceux qui vont à l'église», c'est que, à leurs yeux, l'alcoolisme et la religion représentent, dans le Cameroun contemporain, l'opium du peuple. En faisant référence à la catégorie de «jeunesse inconsciente», ils ciblent également les jeunes qui défendent l'idée de la nécessité, pour les populations de leur zone géographique, de se mobiliser pour lancer un appel à la candidature de Paul

54. République du Cameroun, Pnud, UN-Habitat, Diagnostic de la délinquance urbaine à Yaoundé, Nairobi, UN-Habitat, 2002.

55. D. E. Atangana, «Cameroun: Contre l'appel de la Lékié... », art. cité.

56. La Nouvelle Expression, $\mathrm{n}^{\circ}$ 4183, 10 mars 2016, p. 5.

57. Au moment où nous nous entretenons avec lui, Denis avoue ne plus faire partie du MRC.

58. Entretien avec Denis Atangana, Yaoundé, 13 décembre 2017. 
Biya. Le contre-appel imaginé par ces jeunes de la Lekié revêt une importance capitale car il contredit l'imaginaire politique construit et véhiculé par les élites de ce département, aussi bien au niveau national que local, selon lequel le département est un allié indéfectible du président.

Bien sûr, les jeunes de la Lekié et du pays entier ne se retrouvent pas de façon homogène dans les contre-appels. Ainsi, refusant d'être éclaboussé par ce contre-appel, un jeune leader estudiantin de la Lekié «sera amené59 » à contester la légitimité de ses auteurs à parler «au nom des jeunes ${ }^{60}$ ». Dans son argumentation, il récuse à Denis la qualité de porte-parole des jeunes de la Lekié, lui qui n'a aucune responsabilité au sein de l'Association des étudiants de la Lekié (Aedele), un des cercles majeurs de recrutement et de promotion de l'élite administrative de ce département, encore moins au sein du Conseil départemental de la jeunesse.

\section{Au-delà des jeunes...}

Parmi les porteurs des contre-appels, on compte aussi de jeunes leaders insérés dans des partis d'opposition. Tels sont, par exemple, les cas de Parfait, 41 ans, et de Denis, 29 ans, dont nous avons déjà évoqué les profils. Tel est aussi le cas de Franck Essi, 34 ans, secrétaire général du Cameroon People's Party (CPP). Tous trois assument un leadership et ont en commun d'avoir individuellement initié des contre-appels à la candidature de Paul Biya en 2018. Le Contre-appel national de la jeunesse consciente et républicaine auquel nous avons déjà fait allusion et qu'ils font par la suite le choix d'écrire ensemble manifeste, au-delà des querelles inter-partisanes qui souvent les opposent, leur convergence quant au refus de voir le président de la République se maintenir à la tête de l'État.

Pour autant, il est réducteur de circonscrire le profil des porteurs des contre-appels aux seuls jeunes. C'est ce que tend à accréditer l'initiative de Prosper Nkou Mvondo, promoteur du parti Univers, qui a procédé, le 20 février 2016 à Ngaoundéré, au lancement du mouvement «2018: Tout sauf Paul Biya». Cependant, en parcourant les contre-appels, on se rend compte que les jeunes, quand ils ne sont pas au départ des initiatives, en constituent le thème principal. Deux raisons aident à comprendre cet état de fait. La première a trait à l'enjeu démographique dans la mesure où il est manifeste que les jeunes exercent un ascendant dans la pyramide des âges au sein de la

59. C'est sur le conseil de certaines élites qu'il fera cette sortie. Entretien avec un leader de l'Aedele, membre du RDPC, Yaoundé, 22 décembre 2017.

60. <https://www.youtube.com/watch?v=Bk1JogvSiPY>, consulté le 31 mai 2018. 
société camerounaise. D’après les résultats du troisième recensement général de la population et de l'habitat, les jeunes ayant moins de 25 ans représentent $64,2 \%$ de la population ${ }^{61}$.

Ils constituent donc, et là est la deuxième raison, un gros potentiel pour le marché électoral. D'autant que, autrefois fixé à 21 ans, l'âge légal de vote a été abaissé à 18 ans. Pour entrevoir la portée des contre-appels, il nous faut néanmoins aller au-delà de leur description et de l'analyse du profil de leurs promoteurs.

\section{LES ENJEUX D'UN AFFRONTEMENT}

Deux finalités, au moins, semblent poindre derrière la production des contre-appels. D'une part, une volonté de marketing politique dans le but de conquérir l'opinion. Et, d'autre part, l'ambition de déconstruire la fiction de la naturalité de la candidature de M. Biya à la charge présidentielle.

\section{Conquérir l'opinion}

Pour les porteurs des contre-appels, l'enjeu est de batailler dans l'arène politique pour ne pas laisser le champ libre au pouvoir en place. Pour cela, ils imaginent des initiatives contraires à celles produites par les dignitaires du régime dans un environnement où la mobilisation électorale n'enthousiasme plus vraiment. Bercée par l'idée qu'une des sources possibles de leur faible retentissement dans l'espace politique local résiderait dans leur incapacité à déconstruire l'appréhension, confortée par les appels à candidature, de l'invincibilité du président Paul Biya, cette nouvelle garde des partis d'opposition travaille à combler ce qui, en 2004 et 2011, semble, pour certains d'entre eux, avoir été un déficit stratégique. C'est, du moins, ce qu'indiquent ces paroles de Parfait: «Je me suis rendu compte qu'il y a eu des appels en 2004. En 2011, ...le pouvoir a sorti des tomes. Il y a eu des appels. Et, en 2018, je me suis dit non. Il ne faut pas laisser le pouvoir comme ça. Il faut montrer que c'est pas l'appel du peuple ${ }^{62}$ ».

Dans cet affrontement, d'autres grammaires viennent en appui aux contreappels. À titre illustratif, en 2016, dans l'arrondissement de Yaoundé 4, lors du défilé dédié à la commémoration de la fête de la jeunesse camerounaise,

61. République du Cameroun, La population du Cameroun en 2010, Yaoundé, Bureau central des recensements et des études de la population (Bucrep).

62. Entretien avec Parfait Mbvoum, Yaoundé, 14 décembre 2017. 
des militants du SDF profitent de leur passage devant la tribune où se trouve le sous-préfet pour déployer une banderole à l'intitulé explicite: «La jeunesse de Yaoundé 4 dit non à une nouvelle candidature de Paul Biya en 2018 ou avant». Seulement, l'idée derrière les contre-appels n'est pas seulement de se livrer à une «guerre de l'opinion ${ }^{63}$ ». Il s'agit aussi d'éroder un pan important du socle symbolique sur lequel le régime s'adosse.

\section{Déconstruction symbolique}

En parcourant les contre-appels considérés, on peut systématiser les critiques qui y sont contenues autour de deux principaux points. Elles ciblent, en premier, le président de la République à qui il est explicitement demandé « de prendre sa retraite, après déjà 34 ans à la tête du pays ${ }^{64}$ ». En priant M. Biya de se retirer de la vie politique nationale, les jeunes artisans des contre-appels dénoncent l'inertie politique dont ils jugent qu'il est le premier responsable. Tout comme «Les portes paroles de la Jeunesse consciente et républicaine du Cameroun ${ }^{65}$ » signataires du contre-appel national du même nom, la Jeunesse consciente et républicaine de la Lekié insiste sur les bienfaits qu'un départ à la retraite de celui qui gère le Cameroun depuis plus de 35 ans pourrait engendrer: "permettre à la jeunesse camerounaise de connaître enfin, les joies qu'offre l'alternance politique, apparemment seule forme possible de redistribution intergénérationnelle, dans un environnement hermétique et patrimonialiste ${ }^{66} »$.

La dimension générationnelle est une constante dans les débats politiques locaux. Elle se décline sous deux formes, l'un intra-générationnel et l'autre intergénérationnel. Au sein des générations qui guettent impatiemment «leur tour», les positions divergent. Les vues ne sont en effet pas les mêmes selon qu'on se pense comme ce que j'appellerais une jeunesse de la reproduction, désireuse de perpétuer le régime, ou que, travaillant à renverser le socle politique, économique, social et symbolique sur lequel s'assoit son hégémonie, l'on s'assume comme une jeunesse de la production. Sur le versant intergénérationnel, moins que la catégorie biologique, ce sont les «pathologies» (politiques, sociales et économiques) engendrées par le long séjour à la tête de l'État de M. Biya qui sont énumérées. Le fait qu'il n’aspire pas à se décider

63. Ibid.

64. D. E. Atangana, «Cameroun: Contre l'appel de la Lékié... », art. cité.

65. Le Jour, $\mathrm{n}^{\circ} 2141,10$ mars 2016, p. 8.

66. Ibid. 
Cameroun, l'État stationnaire

librement à aller se reposer préoccupe les porteurs des contre-appels au point que certains lui suggèrent des voies de sortie «honorables»:

«qu'il [Paul Biya] pense à créer une Fondation Politique chargée de promouvoir la démocratie et la paix dans le monde. Ainsi, il restera à coup sûr, visible à jamais et respecté sur le plan international. [...] nous lui proposons de créer la cour constitutionnelle et devenir membre à vie comme en France pour l'ex-Président de la République ${ }^{67}{ }$.

Le propos est sans doute ironique. Il a toutefois le mérite de questionner la phobie de voir son souvenir profané et l'absence d'un dispositif juridique valorisant son statut d'ancien chef d'État comme étant de possibles épées de Damoclès qui justifieraient sa motivation à rester au pouvoir.

En second lieu, «la jeunesse consciente et républicaine» fustige la lâcheté des élites qui, dans ce contexte préélectoral, au lieu de mettre en avant leurs réalisations, se servent du nom du président comme d'un gri-gri politique. Candidat malheureux à l'élection municipale de 2013 dans la ville de Monatélé où il défiait $\mathrm{M}$. Tsala Messi, le maire sortant et candidat du RDPC à sa propre succession, Denis est bien placé pour en parler: «Le RDPC, ils ne font pas les campagnes en mettant les élus devant. Pour eux, c'est toujours Paul Biya. Donc, les populations ont l'impression que Paul Biya, il est le président de la République, il est le maire, il est le député68 ». Dans cette configuration, le nom du président sert de fonds de commerce et d'alibi politiques à des élites qui se défaussent. En plus de l'utiliser comme paravent derrière lequel elles exercent ce qui me semble être un contrôle supérieur de l'État ${ }^{69}$, ce que reprochent les porteurs de ces contre-appels aux élites, c'est leur manque de dévouement à l'égard de leurs «fiefs». Alors qu'elles prétendent travailler pour le bénéfice des populations, elles seraient ainsi davantage préoccupées par leur confort personnel.

Au-delà de l'élection présidentielle de 2018, appels et contre-appels donnent à voir la manière dont s'expriment les tensions entre partisans du statu quo et acteurs qui, parce que souhaitant provoquer l'alternance, défient

67. D. E. Atangana, «Cameroun: Contre l'appel de la Lékié...», art. cité.

68. Entretien avec Denis Atangana, Yaoundé, 13 décembre 2017.

69. L'expression «contrôle supérieur de l'État» fait référence à divers mécanismes qu'imaginent les élites pour garantir leur accès à des positions de pouvoir au sein de la structure étatique. Elle est construite par opposition à celle de «contrôle inférieur de l'État» que nous avons forgée pour désigner «la réappropriation, par le bas, du travail d'audit et de contrôle de la dépense publique effectué par le ministère en charge du contrôle supérieur de l'État». Pour s'en faire une idée plus large, voir J.-M. Manga et A. R. Mbassi, «De la fin des manifestations à la faim de manifester: revendications publiques, rémanence autoritaire et procès de la démocratie au Cameroun ", Politique africaine, $\mathrm{n}^{\circ} 146,2017$, p. 73-97. 
le régime au pouvoir. Par-delà les gains matériels et symboliques escomptés, pour les premiers, les appels à candidature apparaissent comme un outil pour consolider leurs positions. Ils participent, de la sorte, d'une solidarité hégémonique qui, mettant provisoirement sous le boisseau les luttes factionnelles, donne à voir la manière dont s'actualisent des imaginaires politiques nourris par la crainte de lendemains qui déchantent. Redoutant qu'un départ de leur champion entraîne la perte de leurs privilèges, les appels à candidature peuvent aussi être appréhendés comme un moyen pour les élites d'enserrer le prince et de l'acculer à rester au pouvoir. Dans cette perspective, la longévité du président devient une ressource pour conserver certains monopoles. Conscientes de l'embarras que suscite la candidature de M. Biya, ces élites tentent de fabriquer un habillage pour lui donner une légitimité qui viendrait du peuple. Cet artifice est important car il permet au régime d'afficher, aux yeux du monde, son adhésion à l'idéal démocratique perçu comme un élément de civilité politique internationale ${ }^{70}$.

À côté des élites qui travaillent à perpétuer le statu quo, d'autres catégories d'acteurs réclament une alternance au sommet de l'État. Cette volonté de renouvellement s'observe y compris chez certains membres du parti au pouvoir. On repère, par exemple, des lignes de fissures et des rivalités entre partisans d'une modernisation du RDPC et tenants, plus nombreux, d'un conservatisme. Pour autant, c'est chez des challengers évoluant dans l'opposition que percent les voix qui se font le plus entendre. Pour la plupart, on a affaire à de jeunes leaders. Et, quand bien même ils ne le sont pas, ils alertent tous sur le besoin d'un nouveau contrat social où la question générationnelle est perçue comme une urgence. Là n'est néanmoins pas le cœur de leurs exigences. Le départ de Paul Biya et, avec lui, du RDPC, dont certains estiment que la (mal-)gouvernance coûte de plus en plus cher au pays, est au centre de leurs revendications. C'est ce qui les amène à considérer sa longévité et, surtout, les pathologies dont ils estiment qu'elles sont le produit, comme des facteurs paralysants la dynamique de rayonnement du pays. En remettant en cause publiquement le bilan du prince et en s'en prenant à ses lieutenants, des élites vieillissantes pour l'essentiel, les challengers se battent pour un renouvellement accompagné d'un renouveau. Il s'agit d'éroder le socle symbolique du régime en tentant de faire mentir l'hypothèse de la naturalité de sa candidature.

Les pratiques discursives qu'ils produisent et les initiatives qu'ils imaginent nous introduisent au cœur de deux problématiques complémentaires que sont l'alternance politique et le renouvellement de l'opposition. Bien que l'on

70. Y. A. Chouala, «Éthique et politique internationale africaine du XXI ${ }^{\mathrm{e}}$ siècle: les normes de civilité à l'épreuve du jeu réaliste des États », Politiques et sociétés, vol. 25, n 2-3, 2006, p. 183-217. 
observe une asymétrie entre la production des appels et des contre-appels, qu'aggravent leurs écarts de format, de formalisation et de stratégies de publicisation, l'écho des contre-appels est néanmoins amplifié par l'aura de personnalités qui se posent, par les positions qu'elles occupent et les sites de diffusion auxquels elles ont accès, comme des haut-parleurs structurant, parfois, l'agenda de la discussion publique nationale. Si l'un des mérites des contre-appels est de révéler les limites d'un discours qui n'envisage le départ de Paul Biya qu'en lien avec des scénarii apocalyptiques, l'on peut tout de même, se poser la question de savoir s'ils ne profitent pas, in fine, au régime en place dans la mesure où ils contribuent à lui donner une image « démocratique» où la parole, même contestataire, est autorisée

Jean-Marcellin Manga
Laboratoire camerounais d'études et de recherches sur les sociétés contemporaines (Ceresc),
Université de Yaoundé II
Laboratoire d'anthropologie sociale et culturelle (Lasc),
Université de Liège

\section{Abstract}

The People's "Calls" and "Counter-calls" For Paul Biya's Candidature:

Pre-election Confrontation, Hegemonic Tensions and the Struggle for Political Change in Cameroon

This paper questions the "calls" and "counter-calls" for Paul Biya's candidature as a means to participate in political life. The calls for the candidature of the incumbent President show the construction of political allegiances that underlie various clientelist negotiations initiated by political leaders to maintain their hegemonic positions. However, the counter-calls highlight how, through various strategies, their rivals strive to erode the symbolism which, for more than three decades, showcase Paul Biya as the natural candidate to the presidential duty. By focusing on these electoral mobilisation technologies, this article stresses the procedural dimension of elections. Thus, by giving importance to the pre-electoral period rather than to the short electoral operations, the article proposes an original entry point for the study of the conflictual and contradictory dynamics of Cameroon's polity. 